The contribution of diabetes is still widely overlooked in TB prevention efforts [1]. In this sample of Greenland Inuit where both HIV and undernutrition remain uncommon, the risk of developing TB was markedly higher in persons with co-existing diabetes than in persons without. The very high risk associated with diabetes suggests potential reduction in the Greenland TB incidence rates through incorporating diabetes control and prevention in TB prevention schemes. However, the validity of the results should be confirmed in a larger sample size, preferentially by applying longer follow-up time to the study cohort.

\section{Stine Byberg*, Bolette Soborg", Mikael Andersson ", Peter Bjerregaard ${ }^{+}$and Marit E. Jørgensen*}

*Epidemiology group, Steno Diabetes Center A/S, Gentofte, \#Department of Infectious Disease Epidemiology, Statens Serum Institut, "Department of Epidemiology Research, Statens Serum Institut, and ${ }^{+}$Centre of Health Research in Greenland, National Institute of Public Health, University of Southern Denmark, Copenhagen Denmark.

Correspondence: S. Byberg, Epidemiology Group, Steno Diabetes Center A/S, Niels Steensensvej 2-4, DK-2820, Gentofte, Denmark. E-mail: stinebyberg@gmail.com

Statement of Interest: None declared.

Acknowledgements: The present study received funding from the Commission of Scientific Research in Greenland (Nuuk, Greenland). The B99 and IHIT studies were funded by the Danish Medical Research Council and Karen Elise Jensen's Foundation.

\section{REFERENCES}

1 Jeon CY, Murray MB. Diabetes mellitus increases the risk of active tuberculosis: a systematic review of 13 observational studies. PLoS Med 2008; 5: e152.

2 Jørgensen ME, Bjerregaard P, Borch-Johnsen K. Diabetes and impaired glucose tolerance among the inuit population of Greenland. Diabetes Care 2002; 25: 1766-1771.

3 Soborg B, Koch A, Thomsen VØ, et al. Ongoing tuberculosis transmission to children in Greenland. Eur Resp J 2010; 36: 878-884.

4 Bjerregaard P. Greenland survey 2005-2010. Population sample and survey methods. 2nd Edn. www.si-folkesundhed.dk/upload/ inuit_health_in_transition_greenland_methods_5_2nd_revision.pdf Date last updated: 2011. Date last accessed: September 12, 2011.

5 Søborg C, Søborg B, Puoelsen S, et al. Doubling of the tuberculosis incidence in Greenland over an 8-years period (1990-1997). Int J Tuberc Lung Dis 2001; 5: 257-265.

6 Ponce-De-Leon A, Garcia-Garcia MAL, Garcia-Sancho MAC. Tuberculosis and diabetes in Southern Mexico. Diabetes Care 2004; 27: 1584-1590.

7 Mori MA, Leonardson G, Welty TK. The benefits of isoniazid chemoprophylaxis and risk factors for tuberculosis among Oglala Sioux Indians. Arch Intern Med 1992; 152: 550.

8 Ladefoged K, Rendal T, Skifte T, et al. Risk factors for tuberculosis in Greenland: case-control study. Int J Tuberc Lung Dis 2011; 15: 44-49.

9 Leegaard A, Riis A, Kornum JB, et al. Diabetes, glycemic control, and risk of tuberculosis. Diabetes Care 2011; 34: 2530-2535.

10 Søborg B, Andersen AB, Larsen $\mathrm{HK}$, et al. Detecting a low prevalence of latent tuberculosis among health care workers in Denmark detected by $M$. tuberculosis specific IFN- $\gamma$ whole-blood test. Scand J Infect Dis 2007; 39: 554-559.

\title{
Outcomes of a tuberculosis contact investigation programme in Italy
}

\section{To the Editors:}

Mycobacterium tuberculosis transmission is affected by several key factors, such as contagiousness of the index tuberculosis (TB) case, immune status and susceptibility of the exposed TB contact, duration and patterns of contact between the index TB case and the exposed TB contact, and characteristics of the environment within which such contact occurs [1-3]. Tracing strategies that allow the early identification and appropriate treatment of TB contacts with latent $\mathrm{TB}$ infection (LTBI) or active TB should be a priority of TB control programmes with adequate resources $[3,4]$.

In agreement with the guidelines laid out by the Italian Ministry of Health, the Piedmont Region has activated and regularly updated TB contact investigation procedures [5]. We used data from the Piedmont Region TB contact investigation programme to assess the role of selected risk factors for TB infection (TBI) among TB contacts in the city of Turin, Italy. For each suspected or confirmed pulmonary TB case, active contact investigation was conducted among household members, close contacts and regular contacts, defined according to the stone-in-the-pond method [6] Passive investigation was used for occasional contacts [5].

The Piedmont TB notification systems were used to identify pulmonary TB cases between January 2002 and December 2008. TB cases were classified into three categories of contagiousness: 1) sputum smear-positive and culture-positive (acid-fast bacillus positive $(\mathrm{AFB}+))$; 2 ) sputum smear-negative but culturepositive (CULT+); and 3) sputum smear-negative and culturenegative or not examined, i.e. other than defined (OtD). Interviews were conducted with each $\mathrm{TB}$ case to identify $\mathrm{TB}$ contacts, defined as anyone having shared air with an active TB case. All traced TB contacts were screened for active TB or LTBI using the tuberculin skin test (TST). The TST was performed using tuberculin purified protein derivative (5 IU) according to the Mantoux method [3]. TB contacts were classified as infected either if the induration was $\geqslant 5 \mathrm{~mm}$ in diameter regardless of any prior bacille Calmette-Guérin (BCG) vaccination [3] or if clinical, bacteriological or radiological signs or symptoms of manifest disease were present. If the TST was negative, a second 


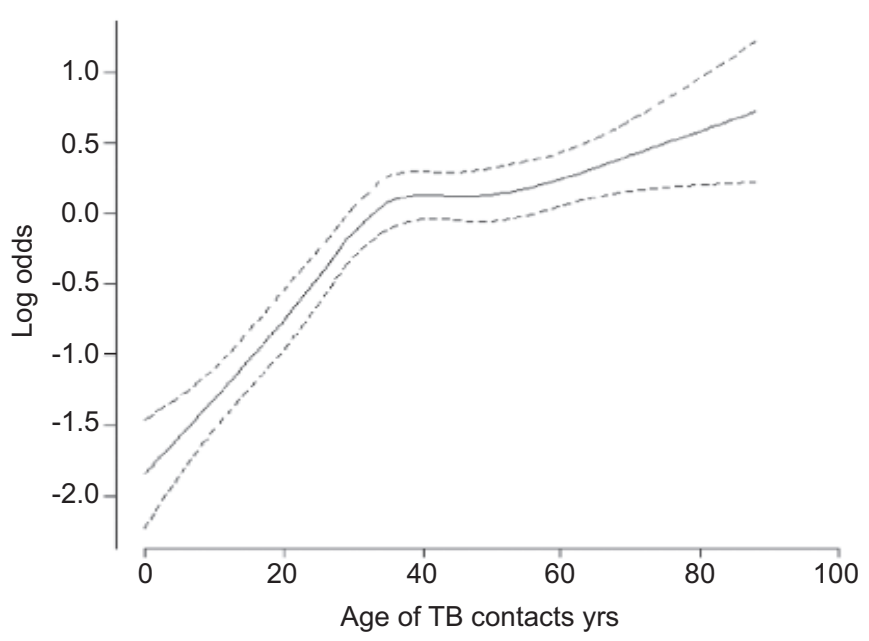

FIGURE 1. Log odds of tuberculin skin test (TST) positivity $(-$ ) with $95 \%$ confidence intervals (- - - ) in evaluated tuberculosis (TB) contacts by age, adjusted for contagiousness, age and sex of the index TB case, and age, sex, place of birth and closeness of contact of TB contacts. TST positivity was defined as tuberculin purified protein derivative reaction of $\geqslant 5 \mathrm{~mm}$.
TST was performed 6-8 weeks later, and that result was considered final and used in the present analyses [5].

$\mathrm{TB}$ contacts were then categorised according to the closeness of contact with the index TB case: household contacts (those living in the same house as the index TB case, e.g. relatives), regular contacts (those who had regular prolonged contact with the index TB case or who spent time with them in a confined space, e.g. friends and colleagues) and occasional contacts (those who shared air with the index TB case for $<12$ h or who spent time with them in an unconfined space) [5].

To account for any expected effect of age on risk of TBI, we tested the nonlinearity of the relationship between age and TST positivity in TB contacts by fitting an unrestricted cubic spline with four knots in a logistic regression model including contagiousness, age and sex of index TB cases, and age, sex, place of birth of TB contacts, and closeness of contact with the index TB case.

Logistic regression was performed in a multilevel analysis to evaluate the predictors of TBI, considering the effect of cluster by TB case. The first level included the characteristics of the index case (contagiousness, age and sex) and the second level included the characteristics of the TB contact (closeness of contact, age, place of birth and sex). We evaluated the statistical

TABLE 1 Results of multilevel analysis for the association between determinants of tuberculosis (TB) infection and active pulmonary $\mathrm{TB}^{\#}$ in evaluated contacts overall, and by age of TB contact

Overall OR $(95 \% \mathrm{Cl})$

Age of TB contacts

\begin{tabular}{|c|c|c|c|}
\hline \multicolumn{2}{|c|}{$\leqslant 35$ yrs } & \multicolumn{2}{|c|}{$>35$ yrs } \\
\hline Subjects $\mathrm{n}$ & OR (95\% Cl) & Subjects $n$ & OR (95\% Cl) \\
\hline
\end{tabular}

\section{First level}

Contagiousness of index TB case

OtD

CULT+

$2.93(1.34-6.41)$

$3.03(1.73-5.32)$

$0.99(0.99-1.00)$

1.00

Age of the index TB case

Males

Females

Second level

Closeness of contact

Occasional

Regular

Household

Age of TB contact

Place of birth of TB contact

Italy

Elsewhere

Sex of TB contact

Male

Female
$0.95(0.75-1.19)$

1.00

$1.08(0.59-1.99)$

$1.53(0.86-2.71)$

$1.05(1.04-1.06)$

$$
\begin{gathered}
1.00 \\
5.22(4.20-6.49) \\
1.00 \\
0.76(0.65-0.90)
\end{gathered}
$$

352
253
1568

1201
972

972

61

345

1767

1296

837

1099

1068
1.00

$2.08(1.09-3.94)$

$2.46(1.54-3.93)$

$0.98(0.97-0.99)$

1.00

$1.01(0.71-1.44)$

1.00

$2.51(0.85-7.40)$

$4.78(1.69-13.50)$

$1.08(1.07-1.10)$

$$
\begin{gathered}
1.00 \\
4.79(3.55-6.47) \\
1.00 \\
0.85(0.67-1.09)
\end{gathered}
$$

340
360
1347

1080
867

867

60

324

1563

1472

395

838

1106
1.00

$0.82(0.53-1.27)$

$1.20(0.87-1.66)$

$1.00(0.99-1.01)$

1.00

$0.98(0.76-1.25)$

1.00

$0.50(0.22-1.14)$

$0.66(0.30-1.43)$

$1.01(1.00-1.02)$

1.00

$4.82(3.47-6.69)$

1.00

$0.64(0.52-0.80)$

OtD: other than defined, i.e. both sputum smear and culture negative or missing, but clinically and/or radiologically diagnosed as being compatible with TB; CULT+: sputum smear examination negative but sputum culture positive for Mycobacterium tuberculosis complex; AFB+: acid-fast bacillus positive, i.e. sputum smear examination positive for acid-fast bacilli and culture positive for $M$. tuberculosis complex. "\#: a positive tuberculin skin test was defined as tuberculin purified protein derivative reaction of $\geqslant 5 \mathrm{~mm}$. 
significance of the interactions between the index TB case and the TB contact characteristics. Odds ratios with $95 \%$ confidence intervals were used as a measure of association.

A total of 833 index TB cases with at least one contact were identified; 4,441 contacts were screened using the TST and 3,942 $(82.8 \%)$ were evaluated. The prevalence of TBI among contacts was $45.0 \%(1,773 \mathrm{~TB}$ contacts had an intradermal reaction $\geqslant 5 \mathrm{~mm}) .28(0.7 \%)$ contacts were found to have active TB.

The nonlinearity test showed that the age, adjusted for contagiousness, age and sex of the index TB case, and age, sex, place of birth and closeness of contact of TB contacts, was positively and strongly associated with TBI in TB contacts until $\sim 35$ yrs of age, after which the log-relative risk increased with a much lower slope (fig. 1). Therefore, we stratified age into two categories, below and above the inflection point at $35 \mathrm{yrs}$ of age ( $\leqslant 35$ and $>35 \mathrm{yrs}$ ).

Results of the multilevel analysis, overall and stratified by age of TB contacts, are shown in table 1. Laboratory and clinical results, which were used as an indicator of contagiousness, were significantly associated with the TBI status of contacts: overall, the contacts of both AFB+ and CULT+ cases had a statistically significantly higher risk of TBI than did the contacts of OtD cases (OR 3.03 (95\% CI 1.73-5.32) and OR 2.93 (95\% CI 1.34$6.41)$, respectively). The strength of the association was greater in the age group $\leqslant 35$ yrs compared with $>35$ yrs (OR $2.46(95 \%$ CI 1.54-3.93) and OR 2.08 (95\% CI 1.09-3.94) in the contacts of $\mathrm{AFB}+$ and CULT+ cases, respectively). Closeness of contact was a predictor of TBI, even though the association was statistically significant only in the age group $\leqslant 35 \mathrm{yrs}$, in which household contacts had a significantly higher risk of TBI than occasional contacts (OR 4.78, 95\% CI 1.69-13.50) (table 1).

As expected, contagiousness and closeness of contact were the major determinants of LTBI and active TB risk [7-9]. However, these factors seemed to play a different role depending on the age of the TB contact. In particular, among TB contacts $\leqslant 35$ yrs of age, household contacts and contacts of AFB+ cases displayed the highest risk of TBI. We also found that the contacts of CULT+ cases had a high risk of infection, despite the fact that CULT+ cases are expected to be less contagious than AFB+ cases due to a lower bacterial load.

In $\mathrm{TB}$ contacts aged $>35 \mathrm{yrs}$, the effects of the main risk factors for tuberculin conversion, contagiousness and closeness of contact, apparently disappear, as confirmed by the significant interaction found between the age of TB contacts and the main determinants of TBI, while the effect of place of birth of TB contacts and the cohort effect persisted. It is possible that in the older age group and in foreign-born TB contacts, incident TBI was hidden by a higher prevalence of previous infections. As a consequence, the likelihood of finding true incident TBI was higher in TB contacts $\leqslant 35$ yrs of age. Furthermore, the apparent protective effect of being born in Italy could be due to a higher prevalence of previous TBI in foreign TB contacts born in countries with high TB prevalence.

The operative meaning of this finding is that a number of TB contacts in the older age group, even if they were candidates for LTBI treatment, did not appear to have new TBI. For this reason, it may be suggested that low priority should be given to the treatment of subjects $>35$ yrs of age. This is particularly true for BCG-vaccinated subjects, and for subjects born in countries with high TB prevalence.

In conclusion, our results suggest that $\mathrm{TB}$ contact investigation programmes should be expanded to include contacts of CULT+ cases and regular TB contacts, especially if an outbreak is demonstrated in household contacts. Finally, due to the observed increased risk of TBI, which is likely to be recent, TB contact investigation efforts should be maximised in the population aged $\leqslant 35$ yrs.

Enrica Migliore $^{*, f}$, Alberto Borraccino ${ }^{\#, f}$, Iacopo Baussano ${ }^{\tau,+}$, Pavilio Piccioni $^{\S}$, Aurelia Carosso ${ }^{\S}$ and Massimiliano Bugiani ${ }^{\S}$ *Cancer Epidemiology Unit, ASO San Giovanni Battista Hospital and CPO Piemonte, "Dept of Public Health Science and Pediatrics, University of Turin, ${ }^{\S}$ Unit of Pneumology, National Local Health - ASL TO2, Turin, and "Cancer Epidemiology Unit, UPO “A. Avogadro" and CPO Piemonte, Novara, Italy. ${ }^{+}$Dept of Infectious Disease Epidemiology, St Mary's Campus, Imperial College, London, UK. ${ }^{\dagger}$ Both authors contributed equally to this work.

Correspondence: A. Borraccino, Dept of Public Health Science and Pediatrics, University of Turin, Via Santena 5bis, 10126 Turin, Italy. E-mail: alberto.borraccino@unito.it

Support Statement: This study was partially supported by the Piedmont Region (Ricerca Sanitaria Finalizzata, years 2002 and 2008).

Statement of Interest: None declared.

\section{REFERENCES}

1 Dye C, Lonnroth K, Jaramillo E, et al. Trends in tuberculosis incidence and their determinants in 134 countries. Bull World Health Organ 2009; 87: 683-691.

2 Baussano I, Nunn P, Williams B, et al. Tuberculosis among health care workers. Emerg Infect Dis 2011; 17: 488-494.

3 Centers for Disease Control. Guidelines for the investigation of contacts of persons with infectious tuberculosis. Recommendations from the National Tuberculosis Controllers Association and CDC. MMWR Recomm Rep 2005; 54: 1-47.

4 British Thorax Society. Control and prevention of tuberculosis in the United Kingdom: code of practice 2000. Joint Tuberculosis Committee of the British Thoracic Society. Thorax 2000; 55: 887-901.

5 Piedmont Region. Protocollo per il controllo della TB umana in Piemonte [Protocol for the control of human TB in Piedmont]. DRG 31/27361. Turin, Regione Piemonte, 1998.

6 Veen J. Microepidemics of tuberculosis: the stone-in-the-pond principle. Tuber Lung Dis 1992; 73: 73-76.

7 Reichler MR, Reves R, Bur S, et al. Evaluation of investigations conducted to detect and prevent transmission of tuberculosis. JAMA 2002; 287: 991-995.

8 Aissa K, Madhi F, Ronsin N, et al. Evaluation of a model for efficient screening of tuberculosis contact subjects. Am J Respir Crit Care Med 2008; 177: 1041-1047.

9 Moran-Mendoza O, Marion SA, Elwood K, et al. Risk factors for developing tuberculosis: a 12-year follow-up of contacts of tuberculosis cases. Int J Tuberc Lung Dis 2010; 14: 1112-1119.

DOI: $10.1183 / 09031936.00047312$ 